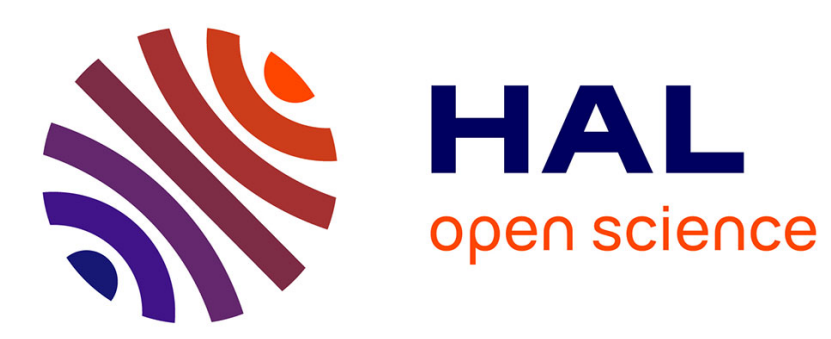

\title{
New quadratic solid-shell elements and their evaluation on linear benchmark problems
}

\author{
Farid Abed-Meraim, Vuong-Dieu Trinh, Alain Combescure
}

\section{To cite this version:}

Farid Abed-Meraim, Vuong-Dieu Trinh, Alain Combescure. New quadratic solid-shell elements and their evaluation on linear benchmark problems. Computing, 2013, 95 (5), pp.373-394. 10.1007/s00607-012-0265-1 . hal-01206680

\section{HAL Id: hal-01206680 \\ https://hal.science/hal-01206680}

Submitted on 29 Sep 2015

HAL is a multi-disciplinary open access archive for the deposit and dissemination of scientific research documents, whether they are published or not. The documents may come from teaching and research institutions in France or abroad, or from public or private research centers.
L'archive ouverte pluridisciplinaire HAL, est destinée au dépôt et à la diffusion de documents scientifiques de niveau recherche, publiés ou non, émanant des établissements d'enseignement et de recherche français ou étrangers, des laboratoires publics ou privés. 


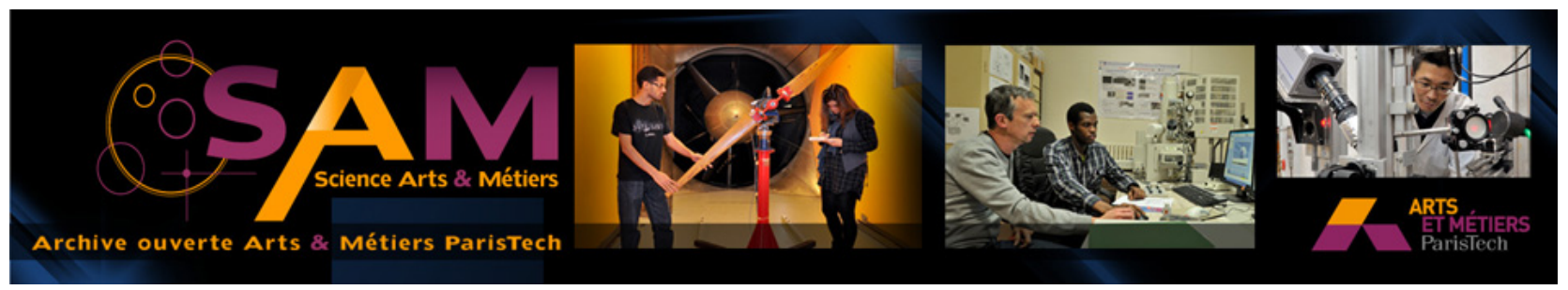

\section{Science Arts \& Métiers (SAM)}

is an open access repository that collects the work of Arts et Métiers ParisTech researchers and makes it freely available over the web where possible.

This is an author-deposited version published in: http://sam.ensam.eu

Handle ID: .http://hdl.handle.net/10985/10228

\section{To cite this version :}

Farid ABED-MERAIM, Vuong-Dieu TRINH, Alain COMBESCURE - New quadratic solid-shell elements and their evaluation on linear benchmark problems - Computing - Vol. 95, n`5, p.373$394-2013$ 


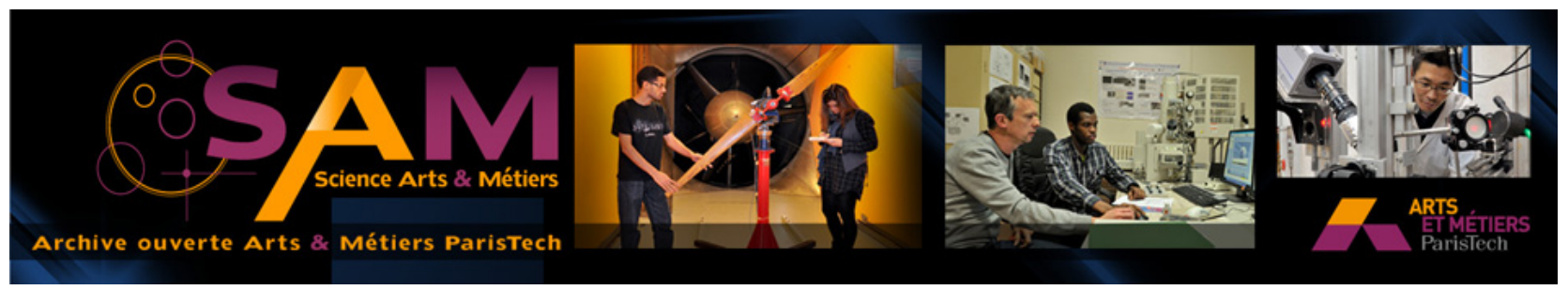

\section{Science Arts \& Métiers (SAM)}

is an open access repository that collects the work of Arts et Métiers ParisTech researchers and makes it freely available over the web where possible.

This is an author-deposited version published in: http://sam.ensam.eu Handle ID: .http://hdl.handle.net/null

\section{To cite this version :}

Farid ABED-MERAIM, Vuong-Dieu TRINH, Alain COMBESCURE - New quadratic solid-shell elements and their evaluation on linear benchmark problems - New quadratic solid-shell elements and their evaluation on linear benchmark problems - Vol. 95, n5, p.373-394 - 2013 


\title{
New quadratic solid-shell elements and their evaluation on linear benchmark problems
}

\begin{abstract}
This paper is concerned with the development of a new family of solidshell finite elements. This concept of solid-shell elements is shown to have a number of attractive computational properties as compared to conventional three-dimensional elements. More specifically, two new solid-shell elements are formulated in this work (a fifteen-node and a twenty-node element) on the basis of a purely three-dimensional approach. The performance of these elements is shown through the analysis of various structural problems. Note that one of their main advantages is to allow complex structural shapes to be simulated without classical problems of connecting zones meshed with different element types. These solid-shell elements have a special direction denoted as the "thickness", along which a set of integration points are located. Reduced integration is also used to prevent some locking phenomena and to increase computational efficiency. Focus will be placed here on linear benchmark problems, where it is shown that these solid-shell elements perform much better than their counterparts, conventional solid elements.
\end{abstract}

F. Abed-Meraim $(\varangle)$

Laboratoire d'Études des Microstructures et de Mécanique des Matériaux, UMR CNRS 7239, Arts et Métiers ParisTech, 4 rue Augustin Fresnel,

57078 Metz Cedex 03, France

e-mail: farid.abed-meraim@ensam.eu

V.-D. Trinh

Laboratoire de Mécanique des Structures Industrielles Durables,

UMR EDF/CNRS/CEA 8193, EDF R\&D, 1 avenue du Général de Gaulle,

92141 Clamart Cedex, France

A. Combescure

Laboratoire de Mécanique des Contacts et des Structures,

UMR CNRS 5259, INSA de Lyon, Bâtiment Jean d'Alembert,

18-20 rue des Sciences, 69621 Villeurbanne Cedex, France 
Keywords Solid-shell finite elements · Mixed variational principle .

Locking phenomena $\cdot$ Reduced integration $\cdot$ Rank deficiency $\cdot$ Benchmark problems

\section{Mathematics Subject Classification $74 \mathrm{~S} 05$}

\section{Introduction}

Nowadays, the numerical simulation at various scales has become widespread in the industry, and concerns practically all key sectors (civil engineering, nuclear, aerospace, automotive, packaging, etc). In computational mechanics, the most adopted approach is certainly the finite element method. Despite the important progress achieved in terms of computer resources, a number of nonlinear three-dimensional (3D) problems still require long hours of calculation times. In this context, the efficiency of finite elements becomes of crucial importance to speed up the design processes, for example, or to reduce the simulation costs in general.

Over the past three decades, considerable effort has been devoted to the development of efficient and reliable finite elements. In this regard, reduced-integration elements have been developed for the efficient treatment of general continuum mechanics problems (see, e.g., [1-9]). Concurrently, for structural applications, a variety of efficient plate and shell elements have been proposed on the basis of mixed formulations or using enhanced assumed strain (EAS) methods in order to avoid locking phenomena (see, e.g., [10-17]).

However, in real-life structures, coexistence of $3 \mathrm{D}$ zones with structural components is very common, and both types of finite element technologies often need to be simultaneously used. Therefore, finite elements that are effective in both thin structure simulation and general 3D applications would considerably simplify the modeling of such combined structures. Indeed, they would avoid arbitrary definitions of separation zones between bulk and structural components, as well as the intricacies related to the connection of different element types (e.g., solid and structural elements).

In addition, 3D element formulations have many other advantages: they avoid resorting to complex shell kinematics, they use general fully 3D constitutive laws, they allow evaluation of thickness variation through the calculation of strain components in that direction, they enable an easier treatment for large rotations and the corresponding configuration update, they make it automatic the connection between solid and structural elements since the displacements are the only degrees of freedom, and allow natural contact conditions on both sides of the structure.

Consequently, significant attention has been paid to the development of a new concept of solid-shell elements, specifically dedicated to the modeling of thin structures (see [18-26]). Most of the methods previously developed were based on enhanced assumed strain fields, and consisted of either the use of a conventional integration rule with appropriate treatment for locking phenomena, or the adoption of a reducedintegration scheme with effective control of zero-energy (hourglass) modes. Both approaches have been extensively investigated and evaluated through a variety of structural applications, as reported in [6,27-30].

A first solid-shell element that we have developed, based on some relatively simple principles, is an eight-node hexahedron denoted as SHB8PS [21,31]. This concept 
aims to combine in a single formulation the well-recognized 3D element advantages with several useful shell features. The evaluation of this element on a variety of benchmark problems confirmed its good performance in terms of accuracy and convergence properties, while using only a single element layer along the thickness. However, with the advent of free mesh generation tools that do not only generate hexahedrons and in order to automatically mesh arbitrarily complex geometries, the development of prismatic solid-shell elements has been made necessary. To this end, a six-node prismatic solid-shell designated as SHB6 has been proposed [32]. Although playing a useful role as a complement to the SHB8PS, the SHB6 element exhibits some shear and thickness-type locking, which is common in linear triangular elements where the strain is constant. The latter limitation represents one of the main motivations behind the current development of alternative complementary solid-shell elements. These proposed elements have quadratic interpolation with both versions, hexahedral and prismatic.

The remaining of the paper is outlined as follows. In Sect. 2, the formulation of the proposed quadratic solid-shell elements, denoted as SHB15 and SHB20, is detailed. In these derivations, the common aspects of the two formulations are emphasized whenever possible. In Sect. 3, the resulting elements are evaluated on a set of selective and representative benchmark problems. Finally, some concluding remarks are drawn in Sect. 4.

\section{Formulation of the SHB15 and SHB20 solid-shells}

In this section, the formulation of the quadratic solid-shell finite elements SHB15 and SHB20 is detailed. Because the adopted approach has a number of similarities for the two elements, both formulations are conjointly presented to avoid repeating the common aspects.

\subsection{Kinematics and interpolation}

The SHB15 and SHB20 denote a fifteen-node prismatic element and a twenty-node hexahedral element, respectively. Based on a 3D approach, they have only three displacement degrees of freedom per node. However, a special direction is chosen, designated as the "thickness", normal to the mean plane of these elements. Also, an in-plane reduced-integration rule is used with $3 \times n_{\text {int }}$ and $4 \times n_{\text {int }}$ integration points for the SHB15 and SHB20, respectively. In other words, the in-plane integration employs only 3 points for the SHB15 and 4 for the SHB20, while the number of throughthickness integration points is $n_{\text {int }}$. Similarly to their linear counterparts SHB8PS and SHB6 [31,32], $n_{\text {int }}$ is a user-defined number, taken equal to two in elasticity and five in plasticity (see Fig. 1).

Note that the integration points for the SHB 15 are located along three lines of respective equations: $(\xi=1 / 2, \eta=1 / 2),(\xi=0, \eta=1 / 2)$, and $(\xi=1 / 2, \eta=0)$, whereas for the SHB20, they are located along the four lines: $(\xi=-1 / \sqrt{3}, \eta=-1 / \sqrt{3}),(\xi=$ $1 / \sqrt{3}, \eta=-1 / \sqrt{3}),(\xi=1 / \sqrt{3}, \eta=1 / \sqrt{3})$, and $(\xi=-1 / \sqrt{3}, \eta=1 / \sqrt{3})$. The 

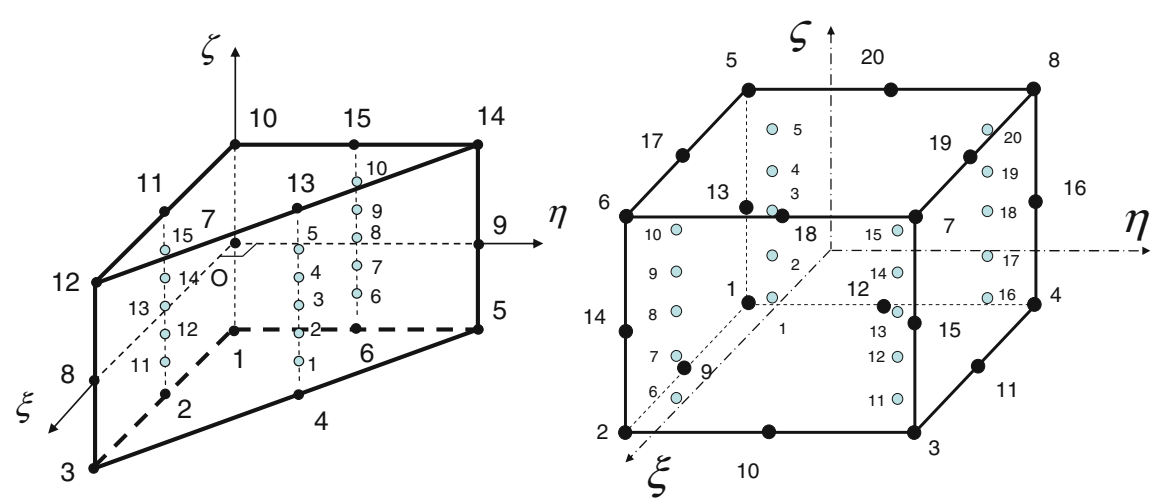

Fig. 1 Reference geometry for the SHB15 and SHB20 and location of integration points $\left(n_{\text {int }}=5\right)$

coordinates of the Gauss points along axis $\zeta$ and the associated weights are given in [33] for the SHB15 and SHB20 for both cases: $n_{\text {int }}=2$ and $n_{\text {int }}=5$.

Within these two isoparametric elements, the spatial coordinates $x_{i}$ and displacement field components $u_{i}$ are related to the nodal coordinates $x_{i I}$ and nodal displacements $u_{i}$, respectively, using the quadratic shape functions $N_{I}$ as

$$
x_{i}=x_{i I} N_{I}(\xi, \eta, \zeta), \quad u_{i}=u_{i I} N_{I}(\xi, \eta, \zeta)
$$

In the equation above and hereafter, unless specified otherwise, the implied convention of summation over repeated indices will be followed. Lowercase subscripts $i$ vary from 1 to 3 and represent the spatial coordinate directions, while uppercase subscripts $I$ vary from 1 to 15 for the SHB15 and from 1 to 20 for the SHB20 and correspond to the element nodes. The explicit expressions of the classical quadratic shape functions $N_{I}$, corresponding to each of the elements SHB15 and SHB20, can be found for instance in [33].

\subsection{Discrete gradient operator}

The interpolation of the displacement field, Eq. (1), will allow us to define the strain field and to express the relationships relating the strain field to the nodal displacements. By differentiating (1), the linear strain tensor is written as

$$
\varepsilon_{i j}=\frac{1}{2}\left(u_{i, j}+u_{j, i}\right)=\frac{1}{2}\left(u_{i I} N_{I, j}+u_{j I} N_{I, i}\right) .
$$

Combining Eq. (1) with the expressions of the shape functions leads to the expansion of the displacement field in the form of a constant term, linear terms in $x_{i}$, and nonlinear terms involving functions $h_{\alpha}$, with $(\alpha=1, \ldots, 11)$ for the SHB15, and $(\alpha=1, \ldots, 16)$ for the SHB20. For the SHB15 element, this expansion reads 


$$
\left\{\begin{aligned}
& u_{i}= a_{0 i}+a_{1 i} x_{1}+a_{2 i} x_{2}+a_{3 i} x_{3}+c_{1 i} h_{1}+c_{2 i} h_{2}+c_{3 i} h_{3}+c_{4 i} h_{4} \\
& \quad+c_{5 i} h_{5}+c_{6 i} h_{6}+c_{7 i} h_{7}+c_{8 i} h_{8}+c_{9 i} h_{9}+c_{10 i} h_{10}+c_{11 i} h_{11}, \quad i=1,2,3 . \\
& h_{1}=\xi \zeta, h_{2}=\eta \zeta, h_{3}=\xi \eta, h_{4}=\xi \eta \zeta, h_{5}=\xi^{2}, h_{6}=\eta^{2}, \\
& h_{7}=\zeta^{2}, h_{8}=\xi^{2} \zeta, h_{9}=\eta^{2} \zeta, h_{10}=\xi \zeta^{2}, h_{11}=\eta \zeta^{2}
\end{aligned}\right.
$$

Evaluating the above equation at the SHB15 element nodes leads to the following three fifteen-equation systems:

$$
\left\{\begin{array}{r}
\mathbf{d}_{i}=a_{0 i} \mathbf{S}+a_{1 i} \mathbf{x}_{1}+a_{2 i} \mathbf{x}_{2}+a_{3 i} \mathbf{x}_{3}+c_{1 i} \mathbf{h}_{1}+c_{2 i} \mathbf{h}_{2}+c_{3 i} \mathbf{h}_{3}+c_{4 i} \mathbf{h}_{4} \\
+c_{5 i} \mathbf{h}_{5}+c_{6 i} \mathbf{h}_{6}+c_{7 i} \mathbf{h}_{7}+c_{8 i} \mathbf{h}_{8}+c_{9 i} \mathbf{h}_{9}+c_{10 i} \mathbf{h}_{10}+c_{11 i} \mathbf{h}_{11}
\end{array}, \quad i=1,2,3\right.
$$

where the fifteen-component vectors $\mathbf{d}_{i}$ and $\mathbf{x}_{i}$ represent the nodal displacements and nodal coordinates, respectively, and are defined as

$$
\mathbf{d}_{i}^{T}=\left(u_{i 1}, u_{i 2}, u_{i 3}, \ldots, u_{i 15}\right), \quad \mathbf{x}_{i}^{T}=\left(x_{i 1}, x_{i 2}, \quad x_{i 3}, \ldots, x_{i 15}\right),
$$

while $\mathbf{s}$ and $\mathbf{h}_{\alpha} \quad(\alpha=1, \ldots, 11)$ are constant vectors given by

$$
\left\{\begin{array}{l}
\mathbf{s}^{T}=(1,1,1,1,1,1,1,1,1,1,1,1,1,1,1) \\
2 \mathbf{h}_{1}^{T}=(0,-1,-2,-1,0,0,0,0,0,0,1,2,1,0,0) \\
2 \mathbf{h}_{2}^{T}=(0,0,0,-1,-2,-1,0,0,0,0,0,0,1,2,1) \\
4 \mathbf{h}_{3}^{T}=(0,0,0,1,0,0,0,0,0,0,0,0,1,0,0) \\
4 \mathbf{h}_{4}^{T}=(0,0,0,-1,0,0,0,0,0,0,0,0,1,0,0) \\
4 \mathbf{h}_{5}^{T}=(0,1,4,1,0,0,0,4,0,0,1,4,1,0,0) \\
4 \mathbf{h}_{6}^{T}=(0,0,0,1,4,1,0,0,4,0,0,0,1,4,1) \\
\mathbf{h}_{7}^{T}=(1,1,1,1,1,1,0,0,0,1,1,1,1,1,1) \\
4 \mathbf{h}_{8}^{T}=(0,-1,-4,-1,0,0,0,0,0,0,1,4,1,0,0) \\
4 \mathbf{h}_{9}^{T}=(0,0,0,-1,-4,-1,0,0,0,0,0,0,1,4,1) \\
2 \mathbf{h}_{10}^{T}=(0,1,2,1,0,0,0,0,0,0,1,2,1,0,0) \\
2 \mathbf{h}_{11}^{T}=(0,0,0,1,2,1,0,0,0,0,0,0,1,2,1)
\end{array}\right.
$$

To determine the unknown constants $a_{j i}$ and $c_{\alpha i}$ in Eq. (2), the derivatives of the shape functions, evaluated at the origin of the reference frame, are introduced:

$$
\mathbf{b}_{i}=\mathbf{N}_{, i}(\mathbf{0})=\frac{\partial \mathbf{N}}{\partial x_{i}}{ }_{\substack{\xi=\eta=\zeta=0\\}} i=1,2,3
$$

where vector $\mathbf{N}$ has as components the shape functions $N_{I}$. Explicit expressions for vectors $\mathbf{b}_{i}$ can be derived by algebra, which allow us to demonstrate the following orthogonality conditions: 


$$
\begin{array}{r}
\mathbf{b}_{i}^{T} \cdot \mathbf{h}_{\alpha}=0, \mathbf{b}_{i}^{T} \cdot \mathbf{s}=0, \mathbf{b}_{i}^{T} \cdot \mathbf{x}_{j}=\delta_{i j}, i, j=1, \ldots, 3 \\
\mathbf{h}_{1}^{T} \cdot \mathbf{s}=0, \mathbf{h}_{2}^{T} \cdot \mathbf{s}=0,2 \mathbf{h}_{3}^{T} \cdot \mathbf{s}=1, \mathbf{h}_{4}^{T} \cdot \mathbf{s}=0, \mathbf{h}_{5}^{T} \cdot \mathbf{s}=4, \mathbf{h}_{6}^{T} \cdot \mathbf{s}=4, \\
\mathbf{h}_{7}^{T} \cdot \mathbf{s}=12, \mathbf{h}_{8}^{T} \cdot \mathbf{s}=0, \mathbf{h}_{9}^{T} \cdot \mathbf{s}=0, \mathbf{h}_{10}^{T} \cdot \mathbf{s}=4, \mathbf{h}_{11}^{T} \cdot \mathbf{s}=4 \\
8 \mathbf{h}_{\alpha}^{T} \cdot \mathbf{h}_{\beta}=\left[\begin{array}{ccccccccccc}
24 & -4 & 0 & 2 & 0 & 0 & 0 & 20 & 2 & 0 & 0 \\
-4 & 24 & 0 & 2 & 0 & 0 & 0 & 2 & 20 & 0 & 0 \\
0 & 0 & 1 & 0 & 1 & 1 & 4 & 0 & 0 & 2 & 2 \\
2 & 2 & 0 & 1 & 0 & 0 & 0 & 1 & 1 & 0 & 0 \\
0 & 0 & 1 & 0 & 26 & 1 & 24 & 0 & 0 & 20 & 2 \\
0 & 0 & 1 & 0 & 1 & 26 & 24 & 0 & 0 & 2 & 20 \\
0 & 0 & 4 & 0 & 24 & 24 & 96 & 0 & 0 & 32 & 32 \\
20 & 2 & 0 & 1 & 0 & 0 & 0 & 18 & 1 & 0 & 0 \\
2 & 20 & 0 & 1 & 0 & 0 & 0 & 1 & 18 & 0 & 0 \\
0 & 0 & 2 & 0 & 20 & 2 & 32 & 0 & 0 & 24 & 4 \\
0 & 0 & 2 & 0 & 2 & 20 & 32 & 0 & 0 & 4 & 24
\end{array}\right]
\end{array}
$$

The constants $a_{j i}$ and $c_{\alpha i}$ can be then determined by the scalar product of (3) by $\mathbf{b}_{j}^{T}, \mathbf{s}^{T}$, and $\mathbf{h}_{\alpha}^{T}$, successively, and the use of orthogonality conditions (5):

$$
a_{j i}=\mathbf{b}_{j}^{T} \cdot \mathbf{d}_{i}, \quad c_{\alpha i}=\boldsymbol{\gamma}_{\alpha}^{T} \cdot \mathbf{d}_{i},
$$

with

$$
\begin{aligned}
\boldsymbol{\gamma}_{\alpha}^{T}= & n_{\alpha 1}\left(\mathbf{h}_{1}^{T}-\left(\mathbf{h}_{1}^{T} \cdot \mathbf{x}_{j}\right) \mathbf{b}_{j}^{T}\right)+n_{\alpha 2}\left(\mathbf{h}_{2}^{T}-\left(\mathbf{h}_{2}^{T} \cdot \mathbf{x}_{j}\right) \mathbf{b}_{j}^{T}\right) \\
& +n_{\alpha 3}\left[\left(\mathbf{h}_{3}^{T}-\frac{1}{30} \mathbf{s}^{T}\right)-\left(\left(\mathbf{h}_{3}^{T}-\frac{1}{30} \mathbf{s}^{T}\right) \cdot \mathbf{x}_{j}\right) \mathbf{b}_{j}^{T}\right] \\
& +n_{\alpha 4}\left(\mathbf{h}_{4}^{T}-\left(\mathbf{h}_{4}^{T} \cdot \mathbf{x}_{j}\right) \mathbf{b}_{j}^{T}\right) \\
& +n_{\alpha 5}\left[\left(\mathbf{h}_{5}^{T}-\frac{4}{15} \mathbf{s}^{T}\right)-\left(\left(\mathbf{h}_{5}^{T}-\frac{4}{15} \mathbf{s}^{T}\right) \cdot \mathbf{x}_{j}\right) \mathbf{b}_{j}^{T}\right] \\
& +n_{\alpha 6}\left(\left(\mathbf{h}_{6}^{T}-\frac{4}{15} \mathbf{s}^{T}\right)-\left(\left(\mathbf{h}_{6}^{T}-\frac{4}{15} \mathbf{s}^{T}\right) \cdot \mathbf{x}_{j}\right) \mathbf{b}_{j}^{T}\right) \\
& +n_{\alpha 7}\left(\left(\mathbf{h}_{7}^{T}-\frac{4}{5} \mathbf{s}^{T}\right)-\left(\left(\mathbf{h}_{7}^{T}-\frac{4}{5} \mathbf{s}^{T}\right) \cdot \mathbf{x}_{j}\right) \mathbf{b}_{j}^{T}\right) \\
& +n_{\alpha 8}\left(\mathbf{h}_{8}^{T}-\left(\mathbf{h}_{8}^{T} \cdot \mathbf{x}_{j}\right) \mathbf{b}_{j}^{T}\right)+n_{\alpha 9}\left(\mathbf{h}_{9}^{T}-\left(\mathbf{h}_{9}^{T} \cdot \mathbf{x}_{j}\right) \mathbf{b}_{j}^{T}\right) \\
& +n_{\alpha 10}\left(\left(\mathbf{h}_{10}^{T}-\frac{4}{15} \mathbf{s}^{T}\right)-\left(\left(\mathbf{h}_{10}^{T}-\frac{4}{15} \mathbf{s}^{T}\right) \cdot \mathbf{x}_{j}\right) \mathbf{b}_{j}^{T}\right) \\
& +n_{\alpha 11}\left(\left(\mathbf{h}_{11}^{T}-\frac{4}{15} \mathbf{s}^{T}\right)-\left(\left(\mathbf{h}_{11}^{T}-\frac{4}{15} \mathbf{s}^{T}\right) \cdot \mathbf{x}_{j}\right) \mathbf{b}_{j}^{T}\right)
\end{aligned}
$$


and

$$
\left[\mathbf{n}_{\alpha \beta}\right]=\left[\begin{array}{ccccccccccc}
\frac{17}{2} & 0 & 0 & -8 & 0 & 0 & 0 & -9 & 0 & 0 & 0 \\
0 & \frac{17}{2} & 0 & -8 & 0 & 0 & 0 & 0 & -9 & 0 & 0 \\
0 & 0 & \frac{256}{17} & 0 & \frac{36}{17} & \frac{36}{17} & 2 & 0 & 0 & -\frac{58}{17} & -\frac{58}{17} \\
-8 & -8 & 0 & 24 & 0 & 0 & 0 & 8 & 8 & 0 & 0 \\
0 & 0 & \frac{36}{17} & 0 & \frac{316}{187} & \frac{146}{187} & 1 & 0 & 0 & -\frac{324}{187} & -\frac{171}{187} \\
0 & 0 & \frac{36}{17} & 0 & \frac{146}{187} & \frac{316}{187} & 1 & 0 & 0 & -\frac{171}{187} & -\frac{324}{187} \\
0 & 0 & 2 & 0 & 1 & 1 & \frac{3}{2} & 0 & 0 & -\frac{3}{2} & -\frac{3}{2} \\
-9 & 0 & 0 & 8 & 0 & 0 & 0 & 10 & 0 & 0 & 0 \\
0 & -9 & 0 & 8 & 0 & 0 & 0 & 0 & 10 & 0 & 0 \\
0 & 0 & -\frac{58}{17} & 0 & -\frac{324}{187} & -\frac{171}{187} & -\frac{3}{2} & 0 & 0 & \frac{505}{187} & \frac{585}{374} \\
0 & 0 & -\frac{58}{17} & 0 & -\frac{171}{187} & -\frac{324}{187} & -\frac{3}{2} & 0 & 0 & \frac{585}{374} & \frac{505}{187}
\end{array}\right]
$$

In the case of the SHB20 element, repeating exactly the same steps results in

$$
\left\{\begin{aligned}
& u_{i}= a_{0 i}+a_{1 i} x_{1}+a_{2 i} x_{2}+a_{3 i} x_{3}+c_{1 i} h_{1}+c_{2 i} h_{2}+c_{3 i} h_{3}+c_{4 i} h_{4}+c_{5 i} h_{5} \\
&+c_{6 i} h_{6}+c_{7 i} h_{7}+c_{8 i} h_{8}+c_{9 i} h_{9}+c_{10 i} h_{10}+c_{11 i} h_{11}+c_{12 i} h_{12}+c_{13 i} h_{13} \\
&+c_{14 i} h_{14}+c_{15 i} h_{15}+c_{16 i} h_{16} \\
& h_{1}=\xi \zeta, h_{2}=\eta \zeta, h_{3}=\xi \eta, h_{4}=\xi^{2}, h_{5}=\eta^{2}, h_{6}=\zeta^{2}, h_{7}=\xi \eta \zeta, h_{8}=\xi^{2} \eta, \\
& h_{9}= \xi^{2} \zeta, h_{10}=\eta^{2} \xi, h_{11}=\eta^{2} \zeta, h_{12}=\zeta^{2} \xi, h_{13}=\zeta^{2} \eta, h_{14}=\xi^{2} \eta \zeta, h_{15}=\xi \eta^{2} \zeta, \\
& h_{16}=\xi \eta \zeta^{2}
\end{aligned}\right.
$$

which, evaluated at the element nodes, yields the three twenty-equation systems:

$$
\begin{gathered}
\mathbf{d}_{i}=a_{0 i} \mathbf{S}+a_{1 i} \mathbf{x}_{1}+a_{2 i} \mathbf{x}_{2}+a_{3 i} \mathbf{x}_{3}+c_{1 i} \mathbf{h}_{1}+c_{2 i} \mathbf{h}_{2}+c_{3 i} \mathbf{h}_{3}+\cdots+c_{16 i} \mathbf{h}_{16}, \\
i=1,2,3 .
\end{gathered}
$$

As before, the twenty-component vectors $\mathbf{d}_{i}$ and $\mathbf{x}_{i}$ indicate here the nodal displacements and coordinates, while $\mathbf{s}$ and $\mathbf{h}_{\alpha}(\alpha=1, \ldots, 16)$ are given by 


$$
\left\{\begin{array}{l}
\mathbf{s}^{T}=(1,1,1,1,1,1,1,1,1,1,1,1,1,1,1,1,1,1,1,1) \\
\mathbf{h}_{1}^{T}=(1,-1,-1,1,-1,1,1,-1,0,-1,0,1,0,0,0,0,0,1,0,-1) \\
\mathbf{h}_{2}^{T}=(1,1,-1,-1,-1,-1,1,1,1,0,-1,0,0,0,0,0,-1,0,1,0) \\
\mathbf{h}_{3}^{T}=(1,-1,1,-1,1,-1,1,-1,0,0,0,0,1,-1,1,-1,0,0,0,0) \\
\mathbf{h}_{4}^{T}=(1,1,1,1,1,1,1,1,0,1,0,1,1,1,1,1,0,1,0,1) \\
\mathbf{h}_{5}^{T}=(1,1,1,1,1,1,1,1,1,0,1,0,1,1,1,1,1,0,1,0) \\
\mathbf{h}_{6}^{T}=(1,1,1,1,1,1,1,1,1,1,1,0,0,0,0,1,1,1,1) \\
\mathbf{h}_{7}^{T}=(-1,1,-1,1,1,-1,1,-1,0,0,0,0,0,0,0,0,0,0,0,0) \\
\mathbf{h}_{8}^{T}=(-1,-1,1,1,-1,-1,1,1,0,0,0,0,-1,-1,1,1,0,0,0,0) \\
\mathbf{h}_{9}^{T}=(-1,-1,-1,-1,1,1,1,1,0,-1,0,-1,0,0,0,0,0,1,0,1) \\
\mathbf{h}_{10}^{T}=(-1,1,1,-1,-1,1,1,-1,0,0,0,0,-1,1,1,-1,0,0,0,0) \\
\mathbf{h}_{11}^{T}=(-1,-1,-1,-1,1,1,1,1,-1,0,-1,0,0,0,0,0,1,0,1,0) \\
\mathbf{h}_{12}^{T}=(-1,1,1,-1,-1,1,1,-1,0,1,0,-1,0,0,0,0,0,1,0,-1) \\
\mathbf{h}_{13}^{T}=(-1,-1,1,1,-1,-1,1,1,-1,0,1,0,0,0,0,0,-1,0,1,0) \\
\mathbf{h}_{14}^{T}=(1,1,-1,-1,-1,-1,1,1,0,0,0,0,0,0,0,0,0,0,0,0) \\
\mathbf{h}_{15}^{T}=(1,-1,-1,1,-1,1,1,-1,0,0,0,0,0,0,0,0,0,0,0,0) \\
\mathbf{h}_{16}^{T}=(1,-1,1,-1,1,-1,1,-1,0,0,0,0,0,0,0,0,0,0,0,0)
\end{array}\right.
$$

In the same way, the introduction of the twenty-component vectors $\mathbf{b}_{i}$, defined by Eq. (4), allows us to demonstrate the following orthogonality conditions:

$$
\begin{gathered}
\mathbf{b}_{i}^{T} \cdot \mathbf{h}_{\alpha}=0, \mathbf{b}_{i}^{T} \cdot \mathbf{s}=0, \mathbf{b}_{i}^{T} \cdot \mathbf{x}_{j}=\delta_{i j}, i, j=1, \ldots, 3 \\
\mathbf{h}_{1}^{T} \cdot \mathbf{s}=0, \mathbf{h}_{2}^{T} \cdot \mathbf{s}=0, \mathbf{h}_{3}^{T} \cdot \mathbf{s}=0, \mathbf{h}_{4}^{T} \cdot \mathbf{s}=16, \mathbf{h}_{5}^{T} \cdot \mathbf{s}=16, \\
\mathbf{h}_{6}^{T} \cdot \mathbf{s}=16, \mathbf{h}_{7}^{T} \cdot \mathbf{s}=0, \mathbf{h}_{8}^{T} \cdot \mathbf{s}=0, \mathbf{h}_{9}^{T} \cdot \mathbf{s}=0, \mathbf{h}_{10}^{T} \cdot \mathbf{s}=0, \\
\mathbf{h}_{11}^{T} \cdot \mathbf{s}=0, \mathbf{h}_{12}^{T} \cdot \mathbf{s}=0, \mathbf{h}_{13}^{T} \cdot \mathbf{s}=0, \mathbf{h}_{14}^{T} \cdot \mathbf{s}=0, \mathbf{h}_{15}^{T} \cdot \mathbf{s}=0, \mathbf{h}_{16}^{T} \cdot \mathbf{s}=0 \\
\mathbf{h}_{\alpha}^{T} \cdot \mathbf{h}_{\beta}=\left[\begin{array}{ccccccccccccccccccc}
12 & 0 & 0 & 0 & 0 & 0 & 0 & 0 & 0 & 0 & 0 & 0 & 0 & 0 & 8 & 0 \\
0 & 12 & 0 & 0 & 0 & 0 & 0 & 0 & 0 & 0 & 0 & 0 & 0 & 8 & 0 & 0 \\
0 & 0 & 12 & 0 & 0 & 0 & 0 & 0 & 0 & 0 & 0 & 0 & 0 & 0 & 0 & 8 \\
0 & 0 & 0 & 16 & 12 & 12 & 0 & 0 & 0 & 0 & 0 & 0 & 0 & 0 & 0 & 0 \\
0 & 0 & 0 & 12 & 16 & 12 & 0 & 0 & 0 & 0 & 0 & 0 & 0 & 0 & 0 & 0 \\
0 & 0 & 0 & 12 & 12 & 16 & 0 & 0 & 0 & 0 & 0 & 0 & 0 & 0 & 0 & 0 \\
0 & 0 & 0 & 0 & 0 & 0 & 8 & 0 & 0 & 0 & 0 & 0 & 0 & 0 & 0 & 0 \\
0 & 0 & 0 & 0 & 0 & 0 & 0 & 12 & 0 & 0 & 0 & 0 & 8 & 0 & 0 & 0 \\
0 & 0 & 0 & 0 & 0 & 0 & 0 & 0 & 12 & 0 & 8 & 0 & 0 & 0 & 0 & 0 \\
0 & 0 & 0 & 0 & 0 & 0 & 0 & 0 & 0 & 12 & 0 & 8 & 0 & 0 & 0 & 0 \\
0 & 0 & 0 & 0 & 0 & 0 & 0 & 0 & 8 & 0 & 12 & 0 & 0 & 0 & 0 & 0 \\
0 & 0 & 0 & 0 & 0 & 0 & 0 & 0 & 0 & 8 & 0 & 12 & 0 & 0 & 0 & 0 \\
0 & 0 & 0 & 0 & 0 & 0 & 0 & 8 & 0 & 0 & 0 & 0 & 12 & 0 & 0 & 0 \\
0 & 8 & 0 & 0 & 0 & 0 & 0 & 0 & 0 & 0 & 0 & 0 & 0 & 8 & 0 & 0 \\
8 & 0 & 0 & 0 & 0 & 0 & 0 & 0 & 0 & 0 & 0 & 0 & 0 & 0 & 8 & 0 \\
0 & 0 & 8 & 0 & 0 & 0 & 0 & 0 & 0 & 0 & 0 & 0 & 0 & 0 & 0 & 8
\end{array}\right]
\end{gathered}
$$


Using the above conditions along with the successive scalar product of (7) by $\mathbf{b}_{j}^{T}, \mathbf{s}^{T}$, and $\mathbf{h}_{\alpha}^{T}$ leads to the same expression (6) for the constants $a_{j i}$ and $c_{\alpha i}$; however, the corresponding vectors $\boldsymbol{\gamma}_{\alpha}$ must be replaced now by

$$
\begin{aligned}
\boldsymbol{\gamma}_{\alpha}^{T}= & n_{\alpha 1}\left(\mathbf{h}_{1}^{T}-\left(\mathbf{h}_{1}^{T} \cdot \mathbf{x}_{j}\right) \mathbf{b}_{j}^{T}\right)+n_{\alpha 2}\left(\mathbf{h}_{2}^{T}-\left(\mathbf{h}_{2}^{T} \cdot \mathbf{x}_{j}\right) \mathbf{b}_{j}^{T}\right) \\
& +n_{\alpha 3}\left(\mathbf{h}_{3}^{T}-\left(\mathbf{h}_{3}^{T} \cdot \mathbf{x}_{j}\right) \mathbf{b}_{j}^{T}\right)+n_{\alpha 4}\left[\left(\mathbf{h}_{4}^{T}-\frac{4}{5} \mathbf{s}^{T}\right)-\left(\left(\mathbf{h}_{4}^{T}-\frac{4}{5} \mathbf{s}^{T}\right) \cdot \mathbf{x}_{j}\right) \mathbf{b}_{j}^{T}\right] \\
& +n_{\alpha 5}\left[\left(\mathbf{h}_{5}^{T}-\frac{4}{5} \mathbf{s}^{T}\right)-\left(\left(\mathbf{h}_{5}^{T}-\frac{4}{5} \mathbf{s}^{T}\right) \cdot \mathbf{x}_{j}\right) \mathbf{b}_{j}^{T}\right] \\
& +n_{\alpha 6}\left[\left(\mathbf{h}_{6}^{T}-\frac{4}{5} \mathbf{s}^{T}\right)-\left(\left(\mathbf{h}_{6}^{T}-\frac{4}{5} \mathbf{s}^{T}\right) \cdot \mathbf{x}_{j}\right) \mathbf{b}_{j}^{T}\right] \\
& +n_{\alpha 7}\left(\mathbf{h}_{7}^{T}-\left(\mathbf{h}_{7}^{T} \cdot \mathbf{x}_{j}\right) \mathbf{b}_{j}^{T}\right)+n_{\alpha 8}\left(\mathbf{h}_{8}^{T}-\left(\mathbf{h}_{8}^{T} \cdot \mathbf{x}_{j}\right) \mathbf{b}_{j}^{T}\right) \\
& +n_{\alpha 9}\left(\mathbf{h}_{9}^{T}-\left(\mathbf{h}_{9}^{T} \cdot \mathbf{x}_{j}\right) \mathbf{b}_{j}^{T}\right)+n_{\alpha 10}\left(\mathbf{h}_{10}^{T}-\left(\mathbf{h}_{10}^{T} \cdot \mathbf{x}_{j}\right) \mathbf{b}_{j}^{T}\right) \\
& +n_{\alpha 11}\left(\mathbf{h}_{11}^{T}-\left(\mathbf{h}_{11}^{T} \cdot \mathbf{x}_{j}\right) \mathbf{b}_{j}^{T}\right)+n_{\alpha 12}\left(\mathbf{h}_{12}^{T}-\left(\mathbf{h}_{12}^{T} \cdot \mathbf{x}_{j}\right) \mathbf{b}_{j}^{T}\right) \\
& +n_{\alpha 13}\left(\mathbf{h}_{13}^{T}-\left(\mathbf{h}_{13}^{T} \cdot \mathbf{x}_{j}\right) \mathbf{b}_{j}^{T}\right)+n_{\alpha 14}\left(\mathbf{h}_{14}^{T}-\left(\mathbf{h}_{14}^{T} \cdot \mathbf{x}_{j}\right) \mathbf{b}_{j}^{T}\right) \\
& +n_{\alpha 15}\left(\mathbf{h}_{15}^{T}-\left(\mathbf{h}_{15}^{T} \cdot \mathbf{x}_{j}\right) \mathbf{b}_{j}^{T}\right)+n_{\alpha 16}\left(\mathbf{h}_{16}^{T}-\left(\mathbf{h}_{16}^{T} \cdot \mathbf{x}_{j}\right) \mathbf{b}_{j}^{T}\right)
\end{aligned}
$$

with

$$
\left[\mathbf{n}_{\alpha \beta}\right]=\left[\begin{array}{cccccccccccccccc}
\frac{1}{4} & 0 & 0 & 0 & 0 & 0 & 0 & 0 & 0 & 0 & 0 & 0 & 0 & 0 & -\frac{1}{4} & 0 \\
0 & \frac{1}{4} & 0 & 0 & 0 & 0 & 0 & 0 & 0 & 0 & 0 & 0 & 0 & -\frac{1}{4} & 0 & 0 \\
0 & 0 & \frac{1}{4} & 0 & 0 & 0 & 0 & 0 & 0 & 0 & 0 & 0 & 0 & 0 & 0 & -\frac{1}{4} \\
0 & 0 & 0 & \frac{3}{8} & \frac{1}{8} & \frac{1}{8} & 0 & 0 & 0 & 0 & 0 & 0 & 0 & 0 & 0 & 0 \\
0 & 0 & 0 & \frac{1}{8} & \frac{3}{8} & \frac{1}{8} & 0 & 0 & 0 & 0 & 0 & 0 & 0 & 0 & 0 & 0 \\
0 & 0 & 0 & \frac{1}{8} & \frac{1}{8} & \frac{3}{8} & 0 & 0 & 0 & 0 & 0 & 0 & 0 & 0 & 0 & 0 \\
0 & 0 & 0 & 0 & 0 & 0 & \frac{1}{8} & 0 & 0 & 0 & 0 & 0 & 0 & 0 & 0 & 0 \\
0 & 0 & 0 & 0 & 0 & 0 & 0 & \frac{3}{20} & 0 & 0 & 0 & 0 & -\frac{1}{10} & 0 & 0 & 0 \\
0 & 0 & 0 & 0 & 0 & 0 & 0 & 0 & \frac{3}{20} & 0 & -\frac{1}{10} & 0 & 0 & 0 & 0 & 0 \\
0 & 0 & 0 & 0 & 0 & 0 & 0 & 0 & 0 & \frac{3}{20} & 0 & -\frac{1}{10} & 0 & 0 & 0 & 0 \\
0 & 0 & 0 & 0 & 0 & 0 & 0 & 0 & -\frac{1}{10} & 0 & \frac{3}{20} & 0 & 0 & 0 & 0 & 0 \\
0 & 0 & 0 & 0 & 0 & 0 & 0 & 0 & 0 & -\frac{1}{10} & 0 & \frac{3}{20} & 0 & 0 & 0 & 0 \\
0 & 0 & 0 & 0 & 0 & 0 & 0 & -\frac{1}{10} & 0 & 0 & 0 & 0 & \frac{3}{20} & 0 & 0 & 0 \\
0 & -\frac{1}{4} & 0 & 0 & 0 & 0 & 0 & 0 & 0 & 0 & 0 & 0 & 0 & \frac{1}{8} & 0 & 0 \\
-\frac{1}{4} & 0 & 0 & 0 & 0 & 0 & 0 & 0 & 0 & 0 & 0 & 0 & 0 & 0 & \frac{1}{8} & 0 \\
0 & 0 & -\frac{1}{4} & 0 & 0 & 0 & 0 & 0 & 0 & 0 & 0 & 0 & 0 & 0 & 0 & \frac{1}{8}
\end{array}\right]
$$
$\alpha, \beta=1,2, \ldots, 16$ 
After these lengthy derivations, the following expressions for the displacement gradient $u_{i, j}$ and for the discrete gradient operator $\mathbf{B}$, which relates the strain field to the nodal displacements by the relationship $\nabla_{s}(\mathbf{u})=\mathbf{B} \cdot \mathbf{d}$, are found:

$$
\begin{aligned}
& u_{i, j}=\left(\mathbf{b}_{j}^{T}+h_{\alpha, j} \boldsymbol{\gamma}_{\alpha}^{T}\right) \cdot \mathbf{d}_{i}, \\
& \mathbf{B}=\left[\begin{array}{ccc}
\mathbf{b}_{x}^{T}+h_{\alpha, x} \boldsymbol{\gamma}_{\alpha}^{T} & \mathbf{0} & \mathbf{0} \\
\mathbf{0} & \mathbf{b}_{y}^{T}+h_{\alpha, y} \boldsymbol{\gamma}_{\alpha}^{T} & \mathbf{0} \\
\mathbf{0} & \mathbf{0} & \mathbf{b}_{z}^{T}+h_{\alpha, z} \boldsymbol{\gamma}_{\alpha}^{T} \\
\mathbf{b}_{y}^{T}+h_{\alpha, y} \boldsymbol{\gamma}_{\alpha}^{T} & \mathbf{b}_{x}^{T}+h_{\alpha, x} \boldsymbol{\gamma}_{\alpha}^{T} & \mathbf{0} \\
\mathbf{0} & \mathbf{b}_{z}^{T}+h_{\alpha, z} \boldsymbol{\gamma}_{\alpha}^{T} & \mathbf{b}_{y}^{T}+h_{\alpha, y} \boldsymbol{\gamma}_{\alpha}^{T} \\
\mathbf{b}_{z}^{T}+h_{\alpha, z} \boldsymbol{\gamma}_{\alpha}^{T} & \mathbf{0} & \mathbf{b}_{x}^{T}+h_{\alpha, x} \boldsymbol{\gamma}_{\alpha}^{T}
\end{array}\right],
\end{aligned}
$$

with

$$
\nabla_{s}(\mathbf{u})=\left[\begin{array}{c}
u_{x, x} \\
u_{y, y} \\
u_{z, z} \\
u_{x, y}+u_{y, x} \\
u_{y, z}+u_{z, y} \\
u_{x, z}+u_{z, x}
\end{array}\right], \quad \mathbf{d}=\left[\begin{array}{c}
\mathbf{d}_{x} \\
\mathbf{d}_{y} \\
\mathbf{d}_{z}
\end{array}\right]
$$

Note that Eqs. (8) and (9) are common to both elements, provided that the summation over subscript $\alpha$ is taken from 1 to 11 for the SHB15, and from 1 to 16 for the SHB20. The compact expression (9) for the discrete gradient operator $\mathbf{B}$ is very convenient because the $\gamma_{\alpha}$ vectors involved are shown to satisfy the following orthogonality conditions:

$\boldsymbol{\gamma}_{\alpha}^{T} \cdot \mathbf{x}_{j}=0, \boldsymbol{\gamma}_{\alpha}^{T} \cdot \mathbf{h}_{\beta}=\delta_{\alpha \beta}, j=1, \ldots, 3,(\alpha, \beta=1, \ldots, 11) \operatorname{resp} .(\alpha, \beta=1, \ldots, 16)$

This would allow each of the deformation modes to be separately handled in order to build a simple form for an assumed-strain field. The formulation (9) also allows us to check any stiffness matrix rank deficiency for these elements. It is noteworthy that no projection for the corresponding discrete gradient operators has been applied to these quadratic solid-shell elements, as no significant locking has been experienced so far in the benchmark problems tested.

\subsection{Variational principle}

The $\mathrm{Hu}-$ Washizu mixed variational principle, which was used for the formulation of the linear solid-shell elements SHB8PS and SHB6 [31,32], is considered here again. More specifically, a simplified form of this principle, as described by Simo and Hughes [34], is adopted, which can be written for a single finite element as 


$$
\pi(\dot{\bar{\varepsilon}})=\int_{\Omega_{e}} \delta \dot{\overline{\boldsymbol{\varepsilon}}}^{T} \cdot \sigma d \Omega-\delta \dot{\mathbf{d}}^{T} \cdot \mathbf{f}^{e x t}=0,
$$

where $\delta$ denotes a variation, $\dot{\bar{\varepsilon}}$ the assumed-strain rate, $\sigma$ the stress field evaluated from the constitutive equations, $\dot{\mathbf{d}}$ the nodal velocities, and $\mathbf{f}^{\text {ext }}$ the external nodal forces. The well-known assumed-strain method consists of choosing a $\overline{\mathbf{B}}$ operator, obtained by projection of the classical discrete gradient operator $\mathbf{B}$, such as $\dot{\bar{\varepsilon}}=\overline{\mathbf{B}} \cdot \dot{\mathbf{d}}$. The goal of this method is to eliminate most of the locking phenomena (e.g., shear locking, membrane locking, etc) that can be encountered, especially in low-order finite elements. Although this technique has been shown to be consistent, from a variational perspective, with the $\mathrm{Hu}$-Washizu variational principle [34], this variational justification does not provide a general, systematic way to derive adequate assumedstrain fields.

Substituting the stress tensor $\boldsymbol{\sigma}$ in Eq. (10) with the Hooke law $\boldsymbol{\sigma}=\mathbf{C} \cdot \overline{\boldsymbol{\varepsilon}}$, where $\mathbf{C}$ is the elasticity matrix, and using the relationship $\dot{\bar{\varepsilon}}=\overline{\mathbf{B}} \cdot \dot{\mathbf{d}}$ yields

$$
\mathbf{K}_{e}=\int_{\Omega_{e}} \overline{\mathbf{B}}^{T} \cdot \mathbf{C} \cdot \overline{\mathbf{B}} d \Omega, \quad \mathbf{f}^{i n t}=\int_{\Omega_{e}} \overline{\mathbf{B}}^{T} \cdot \boldsymbol{\sigma}(\dot{\overline{\boldsymbol{\varepsilon}}}) d \Omega,
$$

where $\mathbf{K}_{e}$ and $\mathbf{f}^{\text {int }}$ denote the stiffness matrix and the internal force vector, respectively.

For the above-mentioned reasons related to the absence of locking for the proposed quadratic solid-shell elements in the benchmark problems tested, no projection for the corresponding discrete gradient operators has been applied here. This amounts to taking $\overline{\mathbf{B}}=\mathbf{B}$ in Eq. (11), which leads to the following classical expressions for the stiffness matrix and internal forces:

$$
\mathbf{K}_{e}=\int_{\Omega_{e}} \mathbf{B}^{T} \cdot \mathbf{C} \cdot \mathbf{B} d \Omega=\sum_{I_{n}=1}^{N_{\mathrm{int}}} \omega\left(\zeta_{I_{n}}\right) J\left(\zeta_{I_{n}}\right) \mathbf{B}^{T}\left(\zeta_{I_{n}}\right) \cdot \mathbf{C} \cdot \mathbf{B}\left(\zeta_{I_{n}}\right),
$$

and

$$
\mathbf{f}^{i n t}=\int_{\Omega_{e}} \mathbf{B}^{T} \cdot \sigma d \Omega=\sum_{I_{n}=1}^{N_{\text {int }}} \omega\left(\zeta_{I_{n}}\right) J\left(\zeta_{I_{n}}\right) \mathbf{B}^{T}\left(\zeta_{I_{n}}\right) \cdot \sigma\left(\zeta_{I_{n}}\right) .
$$

In the above equations, which are common to both developed elements, $J\left(\zeta_{I_{n}}\right)$ is the Jacobian of the transformation between the reference configuration and the current configuration of the element, $\omega\left(\zeta_{I_{n}}\right)$ is the weight associated with the Gauss point $I_{n}$, and the total number of integration points $N_{\text {int }}$ is to adapt depending on the element considered: $N_{\text {int }}=3 \times n_{\text {int }}$ for the SHB15, and $N_{\text {int }}=4 \times n_{\text {int }}$ for the SHB20. 


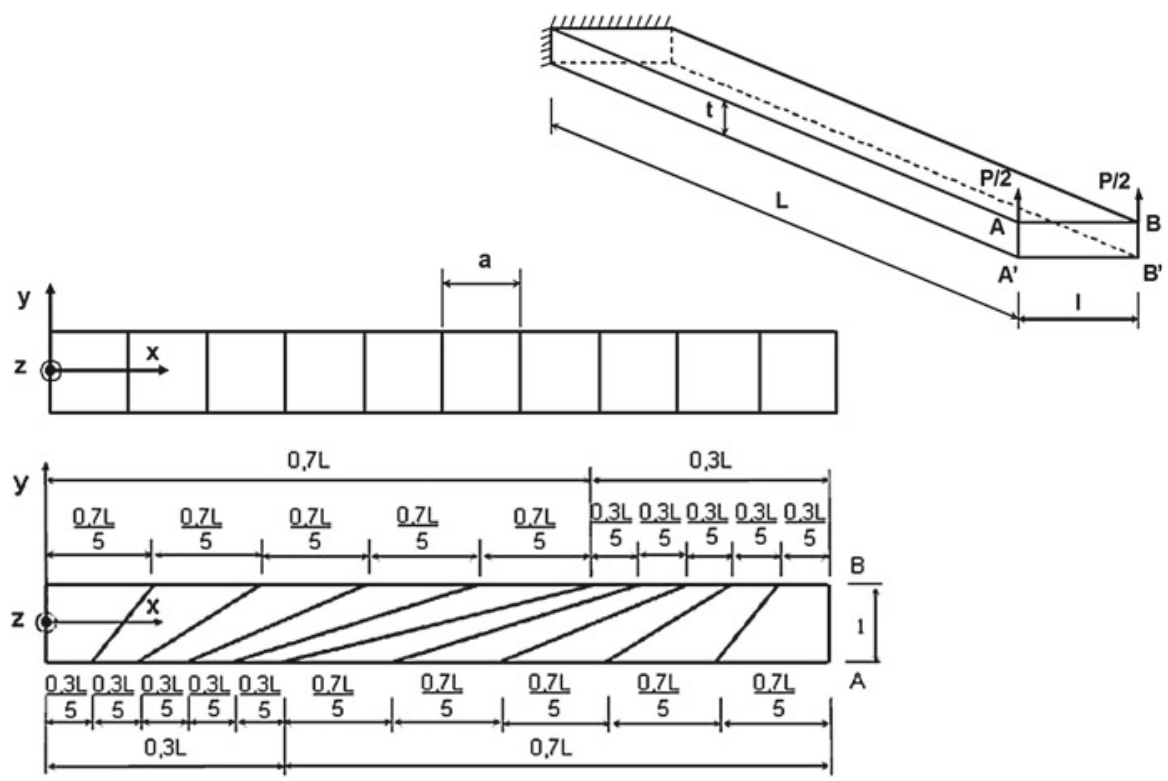

Fig. 2 Test of admissible aspect ratios: Cantilever beam geometry and mesh description

\section{Numerical tests and performance evaluation}

In this section, the performance of the proposed solid-shell elements will be assessed based on the analysis of a set of selective and representative linear benchmark problems. Recall that the formulation of the SHB15 and SHB20 is valid for any number of through-thickness integration points $n_{\text {int }}$, with a minimum number of two. This user-defined number $n_{\text {int }}$ is taken equal to two in linear elastic problems, but can be freely modified whenever required for a specific application. To illustrate the performance of the proposed elements, the results obtained for each test problem will be compared to the reference solution and also to the results yielded by other existing finite elements having similar properties in terms of geometry, number of nodes and degrees of freedom.

\subsection{Test of the maximum aspect ratios}

This linear test is specifically designed to evaluate the aspect ratio limits of the elements on a beam bending problem. It was previously applied to the linear solid-shell SHB8PS [35] in order to assess the behavior of the element when non-structured, irregular meshes are employed and to analyze potential locking phenomena in the limit of high aspect ratios. The corresponding cantilever beam geometry is shown in Fig. 2, where regular and irregular mesh data are specified.

In this analysis, the length and the width of the beam are fixed: $L=100,1=10$; while the thickness $t$ is a varying parameter. The elastic properties are $E=68.25 \times 10^{6}$ 
Table 1 Normalized displacement at point A for the regular mesh

\begin{tabular}{lllll}
\hline Aspect ratio $(\mathrm{r}=1 / \mathrm{t})$ & HEX20 & SHB20 & PRI15 & SHB15 \\
\cline { 2 - 4 } & $u_{z} / u_{z}^{r e f}$ & $u_{z} / u_{z}^{r e f}$ & $u_{z} / u_{z}^{r e f}$ & $u_{z} / u_{z}^{r e f}$ \\
\hline 1 & 1.005 & 1.009 & 1.000 & 1.009 \\
10 & 0.984 & 0.998 & 0.975 & 0.997 \\
100 & 0.951 & 0.997 & 0.967 & 0.995 \\
200 & 0.959 & 0.996 & 0.953 & 1.019 \\
333 & & 0.998 & 0.860 & 0.988 \\
500 & & 1.004 & & \\
\hline
\end{tabular}

Table 2 Normalized displacement at point A for the irregular mesh

\begin{tabular}{lllll}
\hline Aspect ratio $(\mathrm{r}=1 / \mathrm{t})$ & HEX20 & SHB20 & PRI15 & SHB15 \\
\cline { 2 - 5 } & $u_{z} / u_{z}^{r e f}$ & $u_{z} / u_{z}^{r e f}$ & $u_{z} / u_{z}^{r e f}$ & $u_{z} / u_{z}^{r e f}$ \\
\hline 1 & 0.981 & 1.010 & 0.974 & 0.985 \\
10 & 0.682 & 0.997 & 0.871 & 0.945 \\
100 & 0.345 & 0.995 & 0.832 & 0.944 \\
200 & 0.294 & 1.002 & 0.832 & 0.944 \\
333 & 0.251 & 0.984 & 0.878 & 0.926 \\
\hline
\end{tabular}

and $v=0.3$. A bending load, $\mathrm{P}=4$, is applied to the free end of the beam, and the results are normalized using the reference solution, which is here analytical, given by beam theory.

For the hexahedral elements, a fixed mesh of 10 elements with a single element along the thickness is used in both regular and irregular meshes. For the regular mesh, each element is a $10 \times 10$ square (i.e., $1=10$ is the side of the square, and $r=1 / t$ represents the varying aspect ratio). A similar aspect-ratio definition is adopted for the irregular mesh (see Fig. 2). For the prismatic elements, the same procedure as before is followed; then each hexahedron is divided into two prismatic elements, while maintaining a single element layer along the thickness. The normalized vertical displacement at point $A$ for different aspect ratios is reported in Table 1, for the regular mesh, and in Table 2 for the irregular mesh.

In Tables 1 and 2, the proposed solid-shells are compared to their conventional 3D counterparts denoted as PRI15 and HEX20. The latter designate the standard full-integration solid elements with fifteen and twenty nodes, respectively. We can observe that the proposed solid-shells perform much better than their 3D counterparts, especially for the irregular meshes. In this test and for the regular mesh, the admissible aspect ratio is about 500 for the SHB20 and 333 for the SHB15. By contrast, the quality of the results for the standard solid elements starts declining for much lower aspect ratios, and this is more remarkable for distorted meshes (see Table 2). 


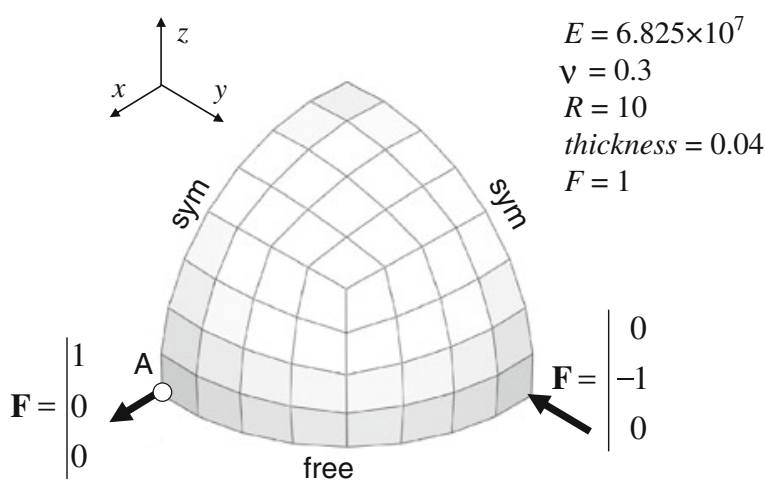

(a)

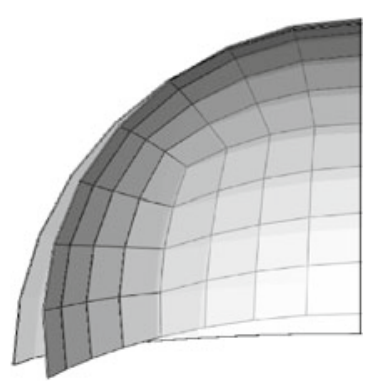

(b)

Fig. 3 Test of the pinched hemispherical shell: a example of a $3 \times(4 \times 4 \times 1)$ mesh, and $\mathbf{b}$ initial and deformed configurations

\subsection{Test of the pinched hemispherical shell}

This doubly-curved shell problem, which is often used to assess the three-dimensional inextensional bending behavior of shells, has become very popular and has been adopted by many authors since it was proposed by MacNeal and Harder [36]. This test is known to be severe because the transverse shear and membrane locking phenomena are dominant and are further accentuated by the particular geometry of the problem (distorted, skewed elements). Figure 3 shows the geometry, loading, and boundary conditions for this elastic thin shell problem $(R / t=250)$. The radius is $R=10$, the thickness is $t=0.04$, Young's modulus is $E=68.25 \times 10^{6}$, and the Poisson ratio is $v=0.3$.

Owing to the symmetry of the test (i.e., planes $(x z)$ and $(y z)$ ), only one quarter of the hemisphere is meshed using a single layer of elements through the thickness and with two unit loads along the directions $O x$ and $O y$. According to the reference solution (MacNeal and Harder [36]), the displacement of point A along the $x$-direction is $u_{x}^{\text {ref }}=0.0924$ (see Fig. 3).

The convergence results are reported in Tables 3 and 4 in terms of normalized displacements at point $A$ in the $x$-direction versus the number of elements. For the hexahedral elements, the mesh nomenclature consists of a $3 \times(N \times N \times 1)$ mesh, in which the quarter of the hemisphere is divided into three zones containing $(N \times N \times 1)$ elements each (see Fig. 3 for an example of a $3 \times(4 \times 4 \times 1)$ mesh). For the prismatic elements, by dividing each hexahedron into two prismatic elements, while maintaining a single element layer through the thickness, the mesh nomenclature becomes $(3 \times$ $(N \times N \times 1)) \times 2$.

The results in Table 3 reveal that the triangular-based elements, SHB15 and PRI15, have a similar convergence in this test problem. Table 4 shows, however, that the quadrangular-based elements converge more rapidly, with a markedly improved performance for the SHB20 as compared to the HEX20. 
Table 3 Normalized displacement at point $\mathrm{A}$ of pinched hemispherical shell (prismatic elements)

Table 4 Normalized displacement at point A of pinched hemispherical shell (hexahedral elements)

\begin{tabular}{lll}
\hline Mesh & PRI15 & SHB15 \\
\cline { 2 - 3 } & $u_{x} / u_{x}^{\text {ref }}$ & $u_{x} / u_{x}^{\text {ref }}$ \\
\hline$(3 \times(5 \times 5 \times 1)) \times 2$ & 0.229 & 0.168 \\
$(3 \times(10 \times 10 \times 1)) \times 2$ & 0.795 & 0.708 \\
$(3 \times(15 \times 15 \times 1)) \times 2$ & 0.954 & 0.934 \\
$(3 \times(20 \times 20 \times 1)) \times 2$ & 0.981 & 0.974 \\
$(3 \times(25 \times 25 \times 1)) \times 2$ & 0.993 & 0.992 \\
\hline
\end{tabular}

\begin{tabular}{lll}
\hline Mesh & HEX20 & SHB20 \\
\cline { 2 - 3 } & $u_{x} / u_{x}^{r e f}$ & $u_{x} / u_{x}^{r e f}$ \\
\hline $3 \times(1 \times 1 \times 1)$ & 0.001 & 0.115 \\
$3 \times(2 \times 2 \times 1)$ & 0.014 & 0.564 \\
$3 \times(3 \times 3 \times 1)$ & 0.065 & 0.927 \\
$3 \times(4 \times 4 \times 1)$ & 0.177 & 0.985 \\
$3 \times(5 \times 5 \times 1)$ & 0.342 & 0.997 \\
\hline
\end{tabular}

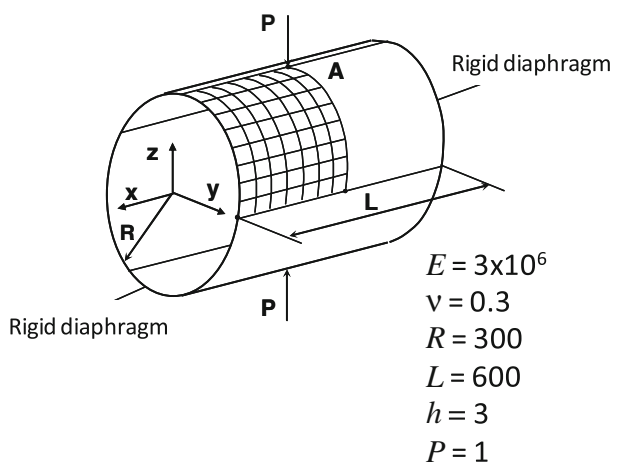

(a)

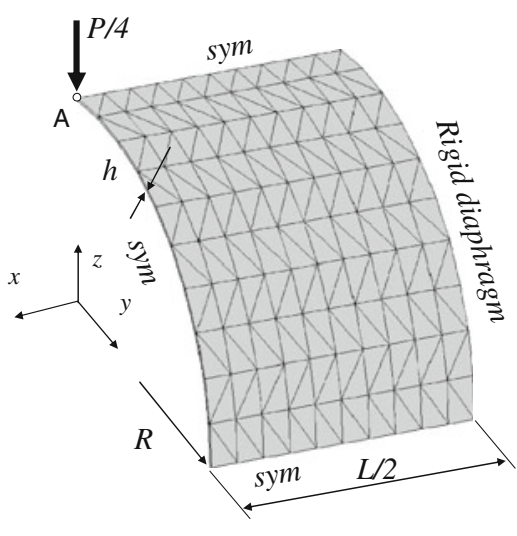

(b)

Fig. 4 Test of the pinched cylinder with diaphragms: a geometry and data specifications, and $\mathbf{b}$ example of a $(10 \times 10 \times 1) \times 2$ mesh with prismatic elements

\subsection{Test of the pinched cylindrical shell with end diaphragms}

A cylindrical shell loaded with a pair of concentrated vertical forces on its middle section is considered here. Both ends of the cylinder are covered with rigid diaphragms that allow displacements only in the axial direction (see Fig. 4). This test has been treated by many authors, among them Belytschko et al. [37] and Chen and $\mathrm{Wu}$ [23]. It is considered as a selective test problem since they have shown that shear locking is more severe than membrane locking. The geometric and material parameters for this problem are described in Fig. 4. 
Table 5 Normalized displacement at point $\mathrm{A}$ of the pinched cylinder (prismatic elements)

\begin{tabular}{lll}
\hline Mesh & PRI15 & SHB15 \\
\cline { 2 - 3 } & $u_{z} / u_{z}^{r e f}$ & $u_{z} / u_{z}^{r e f}$ \\
\hline$(10 \times 10 \times 1) \times 2$ & 0.625 & 0.646 \\
$(15 \times 15 \times 1) \times 2$ & 0.838 & 0.913 \\
$(20 \times 20 \times 1) \times 2$ & 0.919 & 0.951 \\
$(25 \times 25 \times 1) \times 2$ & 0.956 & 0.996 \\
\hline
\end{tabular}

\begin{tabular}{lll}
\hline Mesh & HEX20 & SHB20 \\
\cline { 2 - 3 } & $u_{z} / u_{z}^{r e f}$ & $u_{z} / u_{z}^{r e f}$ \\
\hline $4 \times 4 \times 1$ & 0.140 & 0.883 \\
$6 \times 6 \times 1$ & 0.328 & 0.961 \\
$8 \times 8 \times 1$ & 0.523 & 0.979 \\
$10 \times 10 \times 1$ & 0.675 & 0.990 \\
\hline
\end{tabular}

Table 6 Normalized displacement at point $\mathrm{A}$ of the pinched cylinder (hexahedral elements)

, 


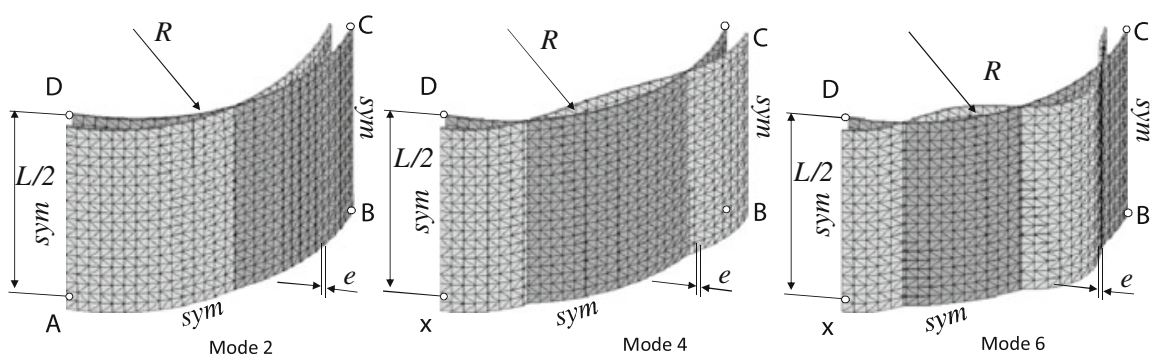

Fig. 5 Buckling of a cylinder under external pressure: buckling modes $n^{0} 2,4$, and 6, and example of a $(20 \times 30 \times 1) \times 2$ mesh with prismatic elements

Table 7 Normalized critical pressure for the thin cylinder under pressure (prismatic elements)

\begin{tabular}{|c|c|c|c|c|c|c|}
\hline \multirow[t]{2}{*}{ Mesh } & \multicolumn{2}{|c|}{$\begin{array}{l}\text { Mode } 2 \\
P_{c r}^{(2)} / P_{c r(r e f)}^{(2)}\end{array}$} & \multicolumn{2}{|c|}{$\begin{array}{l}\text { Mode } 4 \\
P_{c r}^{(4)} / P_{c r(r e f)}^{(4)}\end{array}$} & \multicolumn{2}{|c|}{$\begin{array}{l}\text { Mode } 6 \\
P_{c r}^{(6)} / P_{c r(r e f)}^{(6)}\end{array}$} \\
\hline & PRI15 & SHB15 & PRI15 & SHB15 & PRI15 & SHB15 \\
\hline$(10 \times 10 \times 1) \times 2$ & 1.31 & 1.43 & 1.35 & 1.45 & 1.42 & 1.50 \\
\hline$(12 \times 10 \times 1) \times 2$ & 1.14 & 1.17 & 1.16 & 1.18 & 1.20 & 1.20 \\
\hline$(14 \times 10 \times 1) \times 2$ & 1.07 & 1.08 & 1.08 & 1.08 & 1.11 & 1.09 \\
\hline$(16 \times 10 \times 1) \times 2$ & 1.03 & 1.03 & 1.04 & 1.04 & 1.06 & 1.04 \\
\hline$(20 \times 10 \times 1) \times 2$ & 1.00 & 1.00 & 1.01 & 1.00 & 1.02 & 1.00 \\
\hline
\end{tabular}

in which $\lambda_{c}$ is the critical buckling load and $\mathbf{X}_{c}$ is the associated buckling mode. The geometric and material parameters for this test are (see, e.g., Fig. 5): the length $L=2$, the radius $R=2$, the thickness $e=0.02$, Young's modulus $E=2 \times 10^{11}$, and the Poisson ratio $v=0.3$.

The reference solutions used for comparison are analytical, given by Timoshenko and Gere [38] and Brush and Almroth [39]. Owing to the symmetry, only one eighth of the cylinder is modeled, and symmetry boundary conditions are applied, which in turn restrict the analysis to symmetric buckling modes (i.e., modes 2, 4, and 6 as shown in Fig. 5). The corresponding critical pressure $P_{c r}$ is given by the following analytical expression:

$$
P_{c r}=\frac{E n^{2}}{12\left(1-v^{2}\right)}\left(\frac{e}{R}\right)^{3}, \quad n=2,4,6 .
$$

The results obtained for the three modes ( $n=2,4$, and 6) are reported in Tables 7 and 8 in terms of critical pressure, normalized with respect to the analytical reference solution.

Similar to the previous discussion, Table 7 reveals that the SHB15 exhibits a convergence rate comparable to that of the PRI15 on this test problem. On the contrary, Table 8 shows that the SHB20 has remarkable coarse-mesh accuracy and performs much better than its standard solid counterpart HEX20. 
Table 8 Normalized critical pressure for the thin cylinder under pressure (hexahedral elements)

\begin{tabular}{|c|c|c|c|c|c|c|}
\hline \multirow[t]{2}{*}{ Mesh } & \multicolumn{2}{|c|}{$\begin{array}{l}\text { Mode } 2 \\
P_{c r}^{(2)} / P_{c r(r e f)}^{(2)}\end{array}$} & \multicolumn{2}{|c|}{$\begin{array}{l}\text { Mode } 4 \\
P_{c r}^{(4)} / P_{c r(r e f)}^{(4)}\end{array}$} & \multicolumn{2}{|c|}{$\begin{array}{l}\text { Mode } 6 \\
P_{c r}^{(6)} / P_{c r(r e f)}^{(6)}\end{array}$} \\
\hline & HEX20 & SHB20 & HEX20 & SHB20 & HEX20 & SHB20 \\
\hline $3 \times 3 \times 1$ & 53.33 & 1.00 & 70.58 & 1.05 & 51.73 & 1.26 \\
\hline $4 \times 4 \times 1$ & 17.27 & 0.99 & 21.14 & 1.01 & 25.92 & 1.07 \\
\hline $5 \times 5 \times 1$ & 7.60 & 0.99 & 8.76 & 0.99 & 10.48 & 1.02 \\
\hline $6 \times 6 \times 1$ & 4.16 & 0.98 & 4.61 & 0.99 & 5.31 & 1.00 \\
\hline $7 \times 7 \times 1$ & 2.70 & 0.98 & 2.91 & 0.99 & 3.24 & 0.99 \\
\hline
\end{tabular}

Fig. 6 Geometry and boundary conditions for a quarter of a single ring: an example of a mixed mesh using 260 hexahedral elements for the stiffener and 360 prismatic elements for the main shell

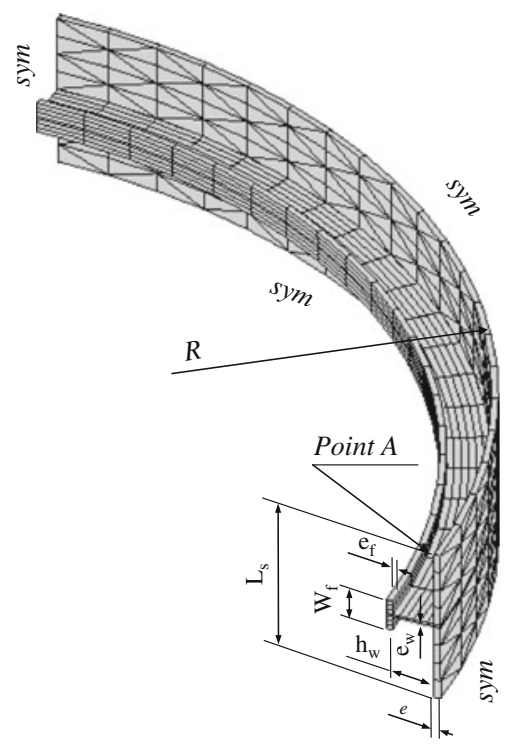

\subsection{Test of buckling of a stiffened cylindrical ring}

This last benchmark problem also deals with linear elastic stability. The corresponding buckling analysis is based on the eigenvalue equation (12), which allows the formulation of the geometric stiffness matrix $\mathbf{K}_{\sigma}$ to be checked once again. The test consists of a portion of a submarine hull subjected to external pressure, as illustrated in Fig. 6.

This test was previously used in [40], while in [41] the submarine was modeled in its entirety. The current analysis only considers a single ring, as described in Fig. 6, and the geometric and material data are reported in Table 9.

Note that due to its symmetry, only one quarter of the ring is modeled and subjected to the corresponding symmetry boundary conditions (see Fig. 6). A linear buckling analysis is performed to determine the Euler critical pressure, based on the eigenvalue analysis of the global stiffness matrix (see Eq. (12)). The SHB8PS element is first used to determine a reference solution for the first critical load (i.e., dead load buckling 
Table 9 Geometric and material properties for the stiffened ring under external pressure

Table 10 Normalized critical buckling pressure for the stiffened cylindrical ring

Fig. 7 Illustration of the first Euler buckling mode for the stiffened cylindrical ring under pressure

\begin{tabular}{lll}
\hline Frame spacing & $L_{s}$ & 0.6 \\
Mean radius & $R$ & 2.488 \\
Shell thickness & $e$ & 0.024 \\
Web height & $h_{w}$ & 0.156 \\
Flange width & $W_{f}$ & 0.120 \\
Web thickness & $e_{w}$ & 0.010 \\
Flange thickness & $e_{f}$ & 0.024 \\
Young's modulus & $E$ & $200 \times 10^{9}$ \\
Poisson's ratio & $v$ & 0.3 \\
\hline
\end{tabular}

\begin{tabular}{ll}
\hline Mesh & $P_{c r} / P_{c r(r e f)}$ \\
\hline 300 PRI15 elements & 1.01 \\
300 SHB15 elements & 1.00 \\
100 HEX20 elements & 1.01 \\
100 SHB20 elements & 0.99 \\
\hline
\end{tabular}

pressure). A converged solution obtained with a fine mesh of 440 SHB8PS elements is $7.06 \times 10^{6}$, which is in good agreement with previously reported results $[40,41]$. The obtained results for the critical pressure, after normalization with respect to the reference solution, are reported in Table 10. The corresponding Euler buckling mode is illustrated in Fig. 7. 
The results in Table 10 reveal, as also observed previously, that the hexahedral solid-shell SHB20 is more efficient in terms of accuracy and convergence than the prismatic solid-shell SHB15.

\section{Conclusions}

In this paper, the formulation of two new quadratic solid-shell finite elements, denoted as SHB15 and SHB20, has been given in detail. The SHB15 is a fifteen-node prism and the SHB20 is a twenty-node hexahedron, both having only three translational degrees of freedom per node. The key idea of the derivation is the convenient combination of a 3D approach with some essential shell features. For instance, the integration points are distributed along a special direction, designated as the thickness. The corresponding in-plane reduced integration contributes to alleviate several locking phenomena, while increasing the computational efficiency of the elements. To further enhance the element immunity with regard to shear and thickness-type locking, shell-like behavior is intended by adopting a local physical coordinate system for the specification of the constitutive matrix. In such a local frame, the $x-y$ plane corresponds to the element mid-plane defined by the $\zeta$-coordinate of the considered integration point.

The performance of the resulting solid-shell elements has been assessed, based on the analysis of various linear benchmark problems. The obtained simulation results have been compared to the reference solutions and also to existing conventional 3D solid elements. In all of the benchmark problems tested, the proposed elements showed very good convergence and accuracy. The performance of the SHB15 solid-shell is often found comparable to that of the standard 3D element PRI15; however, the SHB15 admits larger aspect ratios. The SHB20 solid-shell shows remarkable performance both in terms of coarse-mesh accuracy and computational efficiency. Because these elements have only three degrees of freedom per node, they can automatically be combined to mesh arbitrarily complex structural shapes. This possible combination of effective hexahedral and prismatic elements is useful for many applications, and becomes necessary when free mesh generation tools are employed.

Acknowledgments This work has been carried out within a project jointly funded by EDF R\&D (French Electricity Company) and CETIM (Technical Center for Mechanical Industries). The authors would like to thank EDF R\&D and CETIM for their financial support.

\section{References}

1. Hughes TJR (1980) Generalization of selective integration procedures to anisotropic and nonlinear media. Int J Numer Methods Eng 15:1413-1418

2. Flanagan DP, Belytschko T (1981) A uniform strain hexahedron and quadrilateral with orthogonal hourglass control. Int J Numer Methods Eng 17:679-706

3. Belytschko T, Ong JSJ, Liu WK, Kennedy JM (1984) Hourglass control in linear and nonlinear problems. Comput Methods Appl Mech Eng 43:251-276

4. Liu WK, Hu YK, Belytschko T (1994) Multiple-quadrature underintegrated finite elements. Int J Numer Methods Eng 37:3263-3289

5. Koh BC, Kikuchi N (1987) New improved hourglass control for bilinear and trilinear elements in anisotropic linear elasticity. Comput Methods Appl Mech Eng 65:1-46 
6. Belytschko T, Bindeman LP (1993) Assumed strain stabilization of the eight node hexahedral element. Comput Methods Appl Mech Eng 105:225-260

7. Stainier L, Ponthot JP (1994) An improved one-point integration method for large strain elastoplastic analysis. Comput Methods Appl Mech Eng 118:163-177

8. Hu YK, Nagy LI (1997) A one-point quadrature eight-node brick element with hourglass control. Comput Struct 65:893-902

9. Masud A, Tham CL, Liu WK (2000) A stabilized 3D co-rotational formulation for geometrically nonlinear analysis of multi-layered composite shells. Comput Mech 26:1-12

10. Bathe KJ, Dvorkin EN (1986) Formulation of general shell elements- the use of mixed interpolation of tensorial components. Int J Numer Methods Eng 22:697-722

11. Onate E, Castro J (1992) Derivation of plate based on assumed shear strain fields. In: Ladevèze P, Zienkiewicz OC (eds) New advances in computational structures mechanics. Elsevier, Amsterdam, pp 237-288

12. Cheung Y, Chen WJ (1992) Refined hybrid method for plane isoparametric element using an orthogonal approach. Comput Struct 42:683-694

13. Boisse P, Gelin JC, Daniel JL (1996) Computation of thin structures at large strains and large rotations using a simple $C^{0}$ isoparametric three-node shell element. Comput Struct 58:249-261

14. Ayad R, Dhatt G, Batoz JL (1998) A new hybrid-mixed variational approach for Reissner-Mindlin plates: The MiSP model. Int J Numer Methods Eng 42:1149-1179

15. Chapelle D, Bathe KJ (2003) The finite element analysis of shells_fundamentals. Springer, Berlin

16. Gruttmann F, Wagner W (2004) A stabilized one-point integrated quadrilateral Reissner-Mindlin plate element. Int J Numer Methods Eng 61:2273-2295

17. Cardoso RPR, Yoon JW (2007) One-point quadrature shell elements: a study on convergence and patch tests. Comput Mech 40:871-883

18. Cho C, Park HC, Lee SW (1998) Stability analysis using a geometrically nonlinear assumed strain solid shell element model. Finite Elements Anal Des 29:121-135

19. Hauptmann R, Schweizerhof K (1998) A systematic development of solid-shell element formulations for linear and non-linear analyses employing only displacement degrees of freedom. Int J Numer Methods Eng 42:49-69

20. Sze KY, Yao LQ (2000) A hybrid stress ANS solid-shell element and its generalization for smart structure modelling. Part I-solid-shell element formulation. Int J Numer Methods Eng 48:545-564

21. Abed-Meraim F, Combescure A (2002) SHB8PS - a new adaptive, assumed-strain continuum mechanics shell element for impact analysis. Comput Struct 80:791-803

22. Vu-Quoc L, Tan XG (2003) Optimal solid shells for non-linear analyses of multilayer composites. I. Statics Comput Methods Appl Mech Eng 192:975-1016

23. Chen YI, Wu GY (2004) A mixed 8-node hexahedral element based on the Hu-Washizu principle and the field extrapolation technique. Struct Eng Mech 17:113-140

24. Kim KD, Liu GZ, Han SC (2005) A resultant 8-node solid-shell element for geometrically nonlinear analysis. Comput Mech 35:315-331

25. Klinkel S, Gruttmann F, Wagner W (2006) A robust non-linear solid shell element based on a mixed variational formulation. Comput Methods Appl Mech Eng 195:179-201

26. Reese S (2007) A large deformation solid-shell concept based on reduced integration with hourglass stabilization. Int J Numer Methods Eng 69:1671-1716

27. Zhu YY, Cescotto S (1996) Unified and mixed formulation of the 8-node hexahedral elements by assumed strain method. Comput Methods Appl Mech Eng 129:177-209

28. Wall WA, Bischoff M, Ramm E (2000) A deformation dependent stabilization technique, exemplified by EAS elements at large strains. Comput Methods Appl Mech Eng 188:859-871

29. Puso MA (2000) A highly efficient enhanced assumed strain physically stabilized hexahedral element. Int J Numer Methods Eng 49:1029-1064

30. Soulat D, Cheruet A, Boisse P (2006) Simulation of continuous fibre reinforced thermoplastic forming using a shell finite element with transverse stress. Comput Struct 84:888-903

31. Abed-Meraim F, Combescure A (2009) An improved assumed strain solid-shell element formulation with physical stabilization for geometric nonlinear applications and elastic-plastic stability analysis. Int J Numer Methods Eng 80:1640-1686

32. Trinh VD, Abed-Meraim F, Combescure A (2011) A new assumed strain solid-shell formulation "SHB6" for the six-node prismatic finite element. J Mech Sci Tech 25:2345-2364 
33. Trinh VD (2009) Formulation, développement et validation d'éléments finis de type coques volumiques sous-intégrés stabilisés utilisables pour des problèmes à cinématique et comportement non linéaire. PhD Thesis Dissertation, ENSAM Metz

34. Simo JC, Hughes TJR (1986) On the variational foundations of assumed strain methods. J Appl Mech 53:51-54

35. Legay A, Combescure A (2003) Elastoplastic stability analysis of shells using the physically stabilized finite element SHB8PS. Int J Numer Methods Eng 57:1299-1322

36. MacNeal RH, Harder RL (1985) A proposed standard set of problems to test finite element accuracy. Finite Elements Anal Des 1:3-20

37. Belytschko T, Wong BL, Stolarski H (1989) Assumed strain stabilization procedure for the 9-node Lagrange shell element. Int J Numer Methods Eng 28:385-414

38. Timoshenko SP, Gere JM (1966) Théorie de la stabilité élastique, 2nd edn. Dunod. (Theory of elastic stability). McGraw-Hill, New York

39. Brush DO, Almroth BO (1975) Buckling of bars, plates and shells. McGraw-Hill, New York

40. Legay A (2002) Une méthode de calcul efficace pour l'étude paramétrique du flambage non-linéaire de structures tridimensionnelles : application à la fiabilité. PhD Thesis Dissertation, LMT-ENS-Cachan

41. Bourinet JM, Gayton N, Lemaire M, Combescure A (2000) Reliability analysis of stability of shells based on combined finite element and response surface methods. In: Papadrakis M, Samartin A, Onate E (eds) Computational methods for shell and spatial structures. IASS-IACM, Greece 